\title{
Imaging mechanisms of force detected FMR microscopy
}

\author{
M. M. Midzor ${ }^{\text {a) }}$ \\ Condensed Matter Physics, California Institute of Technology, Pasadena, California 91125 \\ P. E. Wigen \\ Department of Physics, Ohio State University, Columbus, Ohio 43210 \\ D. Pelekhov \\ Los Alamos National Laboratory, Los Alamos, New Mexico 87545 \\ W. Chen \\ Condensed Matter Physics, California Institute of Technology, Pasadena, California 91125 \\ P. C. Hammel \\ Los Alamos National Laboratory, Los Alamos, New Mexico 87545 \\ M. L. Roukes \\ Condensed Matter Physics, California Institute of Technology, Pasadena, California 91125
}

\begin{abstract}
We demonstrate spatial resolution of ferromagnetic resonance in a microscopic sample of YIG using ferromagnetic resonance force microscopy (FMRFM). Measurements were performed on a small single crystal YIG film grown on a GGG substrate, roughly rectangular in shape $20 \mu \mathrm{m} \times \sim 150 \mu \mathrm{m}$ and $3 \mu \mathrm{m}$ thick. The perpendicular and parallel force geometries of FMRFM, in conjunction with an external bias field both parallel and perpendicular to the film, were used to scan the sample. This enabled the detection of strong signals, even at atmospheric pressure and room temperature. The fundamental and higher-order magnetostatic modes were observed to have 26-29 Gauss separation. The intensity of these modes exhibited spatial variation as the magnetic tip was scanned over the sample, and this behavior is qualitatively explained by DE theory. An improved fabrication method for magnet on cantilever was employed, which yielded a spatial resolution of $15 \mu \mathrm{m}$. These results demonstrate the potential of FMRFM for investigating the spatial dependence of ferromagnetic resonance, and for studying the anisotropy fields and exchange coupling effects within multilayer films and small magnetic systems. (C) 2000 American Institute of Physics.
\end{abstract}

[S0021-8979(00)80608-8]

\section{INTRODUCTION}

FMR is an important method for characterizing magnetic materials and devices, especially in the studies of magnetic multilayer systems and magnetic dots. However, in conventional FMR, sensitivity is limited and requires large sample areas $\left(\sim \mathrm{mm}^{2}\right)$. Furthermore, it is generally not possible to characterize, on a microscopic scale, the spatial dependence of magnetic anisotropy and exchange energies in magnetic devices. Recent work ${ }^{1,2}$ has demonstrated the feasibility of performing FMR on microscopic samples using the magnetic resonance force microscope (MREM), but the attainable spatial resolution - and the underlying physical mechanisms that determine it - have not yet been established in microscopic ferromagnetic systems. In this article, the ability of MRFM to detect the spatial dependence of FMR magnetostatic modes in a small YIG sample is demonstrated. An improved method of fabricating a gradient magnet on the cantilever is also described.

In MRFM, magnetic resonance is detected mechanically by coupling the large field gradient from the cantilever magnetic, $\nabla H$, to the spin magnetization in the sample, $\vec{M}$, resulting in a force on the cantilever, $F=\vec{M} \cdot \vec{\nabla} H$. In conventional magnetic resonance imaging (MRI), the field gradient

${ }^{a)}$ Electronic mail: midzor@its.caltch.edu determines a sensitive slice in which the resonance condition, a local function of the applied field, is met. However, in magnetically ordered systems, strong magnetic coupling leads to excitations that are extended throughout the sample, and conditions for these resonances can be strongly influenced by $\vec{H}_{\text {total }}$, the total vector field resulting from the applied bias and gradient magnetic fields. The interaction between the ferromagnetic tip and the sample can locally alter resonance line widths and shapes in a nontrivial manner. In the initial experiments described here, we defer systematic investigation of such dependencies and simply approximate the gradient magnet as a dipole, which yields spatial resolutions comparable to the dimensions of the detection magnet.

\section{EXPERIMENTAL DETAILS}

The MRFM apparatus, as shown in Fig. 1, represents an improvement over our earlier setups. ${ }^{1,2}$ Here the sample is mounted directly upon the microstrip resonator. ${ }^{1}$ The detection cantilever, to which is affixed a small gradient magnet as described below, is scanned over the sample. Measurements were performed on a small single crystal YIG film that is approximately rectangular in shape, $20 \mu \mathrm{m} \times \sim 150 \mu \mathrm{m}$ and 3 $\mu \mathrm{m}$ thick, with a bias field $H$ that could be aligned both perpendicular and parallel to the plane of the YIG film, with the rf field at $7.74 \mathrm{GHz}$. 


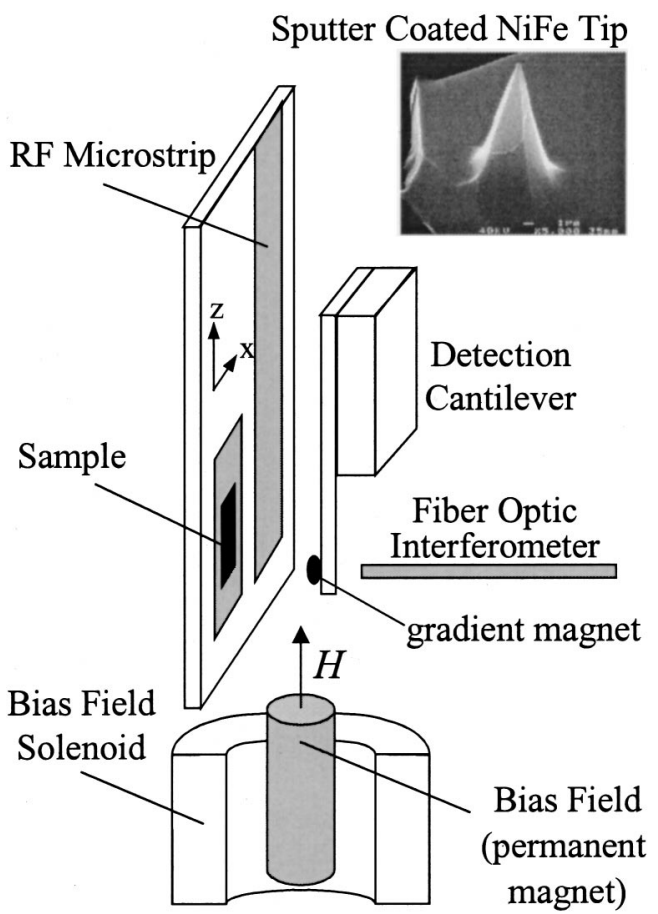

FIG. 1. Schematic of the FMRFM setup (not to scale). An external field, $H$, parallel to the sample plane, is produced by a permanent magnet and swept by the current in a solenoid. The microstrip produces a rf field perpendicular to the applied field, $H$. The inset shows the tip of a commercial cantilever that has been locally sputter coated with $\mathrm{NiFe}$.

An improved detection cantilever (used for $H \perp$ YIG film) was developed using a commercial AFM cantilever ${ }^{3}$ $\left(f_{c} \sim 18 \mathrm{kHz}\right)$ onto which $1200 \AA \mathrm{NiFe}$ (from a $\mathrm{Ni}_{89} \mathrm{Fe}_{19}$ target) was sputter deposited solely in the region of the ultrasharp scanning tip, which was approximately $10 \mu \mathrm{m}$ tall (Fig. 1, inset). A second type of detection cantilever (used for $H \|$ YIG film) consisted of a wedge-shaped NdFeB magnetic particle, approximately $50 \mu \mathrm{m} \times 20 \mu \mathrm{m} \times 15 \mu \mathrm{m}$, glued to the end of a commercial AFM cantilever $\left(f_{c} \sim 8 \mathrm{kHz}\right)$ and prepolarized in a $8 \mathrm{~T}$ field along the direction of $H$.

The force imposed on the detection cantilever is modulated at $f_{c}$ using anharmonic modulation. ${ }^{4}$ In this technique, the bias field $H$ and the rf field $H_{1}$ are separately modulated such that the difference in their modulation frequencies equals $f_{c}$. At resonance, the nonlinear magnetic response imparts a time-varying force on the cantilever, which is mechanically "amplified" by its $Q$. All experiments were performed at room temperature and atmospheric pressure.

\section{RESULTS AND DISCUSSION}

\section{A. $H \|$ YIG film}

In this geometry (Fig. 1), $H$ is aligned parallel to the film plane (directed along the $150 \mu \mathrm{m}$ length of the sample) and to the long axis of the NdFeB particle, and $F=m \cdot \partial H_{Z} / \partial y$ ("perpendicular force geometry," 1 i.e., the induced force and cantilever motion are perpendicular to $H$ ). The external field was greater than $2 \mathrm{kG}$, ensuring that both the sample and gradient magnet were saturated. In this field configuration higher-order modes are expected to occur upfield from the fundamental mode, ${ }^{5}$ which was indeed observed. Figure

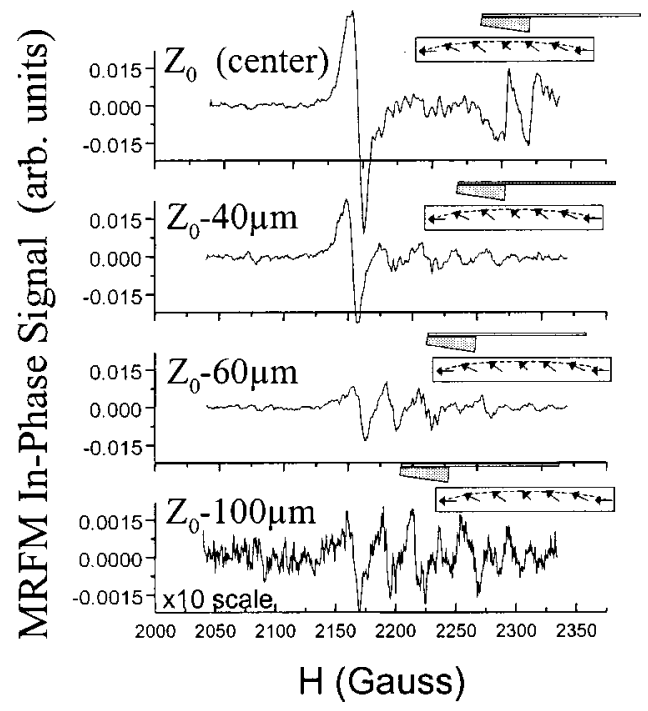

FIG. 2. The MRFM signal obtained from a small YIG film, $H \|$ film plane, as the cantilever is scanned from the center to the edge along the $150 \mu \mathrm{m}$ length.

2 shows the evolution of the MRFM signal as the detection cantilever was scanned from the center to the edge of the YIG film. The largest feature, the fundamental mode, was maximal when the gradient magnet was positioned over the center of the sample, decreased to a minimum at the sample's edges, then vanished when the gradient magnet was far from the sample.

The smaller features correspond to higher-order magnetostatic modes, that were first modeled by Damon and Eshbach $^{6}(\mathrm{DE})$ for infinite thin films, and later calculated by Storey et $\mathrm{al}^{7}$ for the case of thin, finite YIG films. These modes correspond to discrete standing wave vectors $k_{z}$ and $k_{x}$ ( $\hat{z}$ and $\hat{x}$ in the plane of the film), which determine the spatial variation of the magnetization and have wavelengths, $\lambda$, on the order of the linear dimensions of the sample.

Samples with finite dimension $l_{z}$ and $l_{x}$ (with $l_{z}, l_{x} \gg$ thickness) lead to quantized values of $k_{z}$ and $k_{x}$ due to the boundary conditions, $\left(n_{z} \pi / l_{z}\right)$ and $\left(n_{x} \pi / l_{x}\right)$, respectively. For $H \|$ film, the resonance condition can be approximated by $^{8}$

$$
\frac{\omega}{\gamma} \approx H_{\mathrm{ext}}+2 \pi M_{S}-\pi M_{S} d\left[\left(\frac{n_{z} \pi}{L}\right)^{2}+\left(\frac{n_{x} \pi}{w}\right)^{2}\right]^{1 / 2},
$$

where $\gamma=2.8 \mathrm{MHz} / \mathrm{G}$ for YIG, $M_{0}$ is the magnetization, and $L, w$, and $d$ are the length, width, and thickness of the sample. The effect of uniaxial anisotropy is neglected since it does not alter the spacing of the modes, but solely introduces a shift in the resonance frequency of all modes. ${ }^{5}$ The best fit to the data gives $4 \pi M_{0}=1.4 \mathrm{kG}$, rather than the bulk value of $1.8 \mathrm{kG}$, which we attribute to the magnetic anisotropy energy and the deviation of the film from an infinite sheet due to its limited aspect ratio, i.e., the demagnetization factor $N_{Z Z} \neq 4 \pi$.

The measured mode separations are 26-29 G, and the predicted $k_{z}$ separations for the fundamental, third, and fifth modes along the sample's $150 \mu \mathrm{m}$ length $\left(k_{1}, k_{3}, k_{5}\right)$ are 13 , 23 , and $31 \mathrm{G}$ [Eq. (1)]. The even $k_{z}$ modes do not couple to the rf field due to absorption rules. ${ }^{5}$ The difference between 


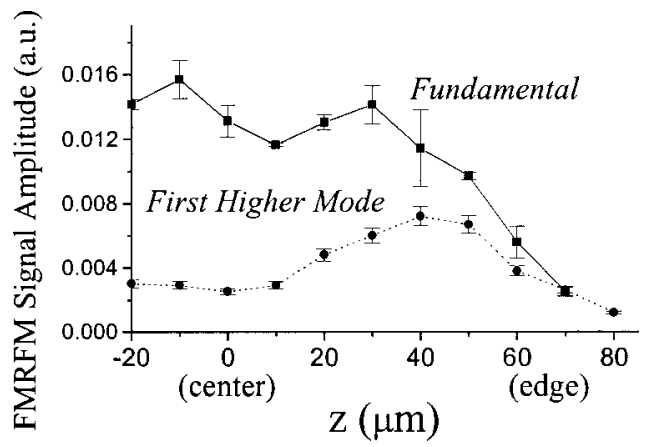

FIG. 3. The amplitude of the fundamental mode and the first higher-order mode of the FMRFM spectra of the YIG sample, as the cantilever is scanned along the $150 \mu \mathrm{m}$ length of the film. The $H \perp$ film plane.

the predicted and observed separations can be accounted for by the inhomogeneity in the internal field due to the relatively small aspect ratio of the sample. The measured mode separations clearly do not correspond to the $20 \mu \mathrm{m}$ dimension, since for this case $k_{x}$ modes separations will be of order of $300 \mathrm{G}$.

The $50 \mu \mathrm{m}$ length of the NdFeB particle limited spatial resolution of the FMR mode to those having a comparable or larger length scale. A tip-to-sample separation of $20 \mu \mathrm{m}$ was used. Estimates of the expected resolution (based upon the $\mathrm{NdFeB}$ particle dimensions) were consistent with the observed variation of the MRFM signal as the particle was moved away from the sample surface; the signal intensity was observed to drop by half at a distance of $18 \mu \mathrm{m}$, consistent with the $15 \mu \mathrm{m} \mathrm{NdFeB}$ particle thickness. Only the main mode exhibited significant amplitude variation as the cantilever was scanned across the $150 \mu \mathrm{m}$ length of the sample. Higher-order modes have significant spatial variation within the $50 \mu \mathrm{m}$ scale of the particle hence, as expected, their contribution to the variation in the FMR signal intensity tends to be suppressed by the inherent spatial averaging. Accordingly, the higher-order signals exhibit rather weak spatial dependence.

\section{B. $H \perp$ YIG film}

In this geometry $H$ is aligned perpendicular to the film, and parallel to the long axis of the NiFe-coated cantilever tip; for this case $F=m \cdot \partial H_{Z} / \partial Z$ ("parallel force geometry"). The external magnetic field was greater than $4 \mathrm{kG}$, again ensuring that the sample and gradient magnet were saturated. The fundamental mode occurs at much higher magnetic fields than in the parallel configuration due to compensation for the demagnetization field of the film, and the higher-order modes occur downfield from the fundamental mode. ${ }^{5}$ Both characteristics were observed. The detection cantilever was scanned from the center to the edge of the YIG film along its longest $(150 \mu \mathrm{m})$ dimension. As before, the largest feature, the fundamental mode, was maximal with the gradient magnet positioned over the center of sample, decreased to a minimum at the sample's edges, and then vanished when the gradient magnet was moved past the sample edge (Fig. 3). The cantilever with the NiFe-coated tip, which demonstrated a spatial resolution of $\sim 15 \mu \mathrm{m}$, greatly improved the sensitivity. Thus, amplitude variations in the fundamental mode, as well as those in several higherorder modes, were resolved as the cantilever was scanned across the sample. For the first higher-order mode, the position of its amplitude maxima occurs toward the edge of the sample; its wavelength, shorter than the fundamental, produces a peak in the mode amplitude closer to the edge of the sample (Fig. 3). At the center of the film the modes are $\sim 27$ $\mathrm{G}$ apart, and their relative amplitudes decrease as mode number increases-behavior that follows the predictions of DE theory.

In this configuration scanning was typically performed $10-15 \mu \mathrm{m}$ above the surface. The estimated resolution of 15 $\mu \mathrm{m}$ was consistent with scans across the width of the sample; the signal strength was found to decrease by half within $15 \mu \mathrm{m}$ of the sample center. Further confirmation was obtained by raising the cantilever from the surface of the sample; in this case the signal strength decreased by half when the cantilever tip was $15 \mu \mathrm{m}$ from the surface.

The NiFe-tipped cantilevers have several important features that are worthy of mention. They can be fabricated consistently with known dimensions and magnetic material thickness, thus increasing the accuracy of gradient field approximations. In the $H \perp$ film configuration, the NiFe tip was saturated, which maximizes (and stabilizes) the gradient field for our scan heights $(\sim 3 \mathrm{G} / \mu \mathrm{m})$. Since the FMR spatial resolution obtained depends on the shape of the tip and its proximity to the sample (i.e., gradient field strength), the $0.1-0.3$ $\mu \mathrm{m}$ tip has the potential to scan with resolution at the micron scale.

\section{CONCLUSIONS}

Spatial variations in the amplitudes of the magnetostatic modes of a microscopic YIG sample have been observed. These can be qualitatively understood using DE theory. A new method of fabricating small, high-gradient-field magnets on commercial cantilevers was developed; these have yielded $15 \mu \mathrm{m}$ spatial resolution in this study of a ferromagnetic sample. This further demonstrates the potential of MRFM to measure microscopic spatial variations in important properties of thin ferromagnetic films, such as interface quality and interlayer exchange coupling.

\section{ACKNOWLEDGMENT}

We gratefully acknowledge funding for this work from the U.S. Department of Energy, Office of Basic Energy Services.

${ }^{1}$ Z. Zhang, P. C. Hammel, M. M. Midzor, M. L. Roukes, and J. R. Childress, Appl. Phys. Lett. 73, 2036 (1998).

${ }^{2}$ Z. Zhang, P. C. Hammel, and P. E. Wigen, Appl. Phys. Lett. 68, 2005 (1996).

${ }^{3}$ SC12 Contact Ultrasharp silicon cantilevers, ND-MDT, P.O. Box 50 103305 Moscow, Russia.

${ }^{4}$ K. J. Bruland, J. Krzystek, J. L. Garbini, and J. A. Sidles, Rev. Sci. Instrum. 66, 2853 (1995).

${ }^{5}$ P. E. Wigen, Thin Solid Films 114, 135 (1984).

${ }^{6}$ R. W. Damon and J. R. Eshbach, J. Phys. Chem. Solids 19, 308 (1961).

${ }^{7}$ B. E. Storey, A. O. Tooke, A. P. Cracknell, and J. A. Przystawa, J. Phys. C 10, 875 (1997).

${ }^{8}$ This is obtained from the Near Fundamental Resonance approximation; M. J. Hurben and C. E. Patton, J. Magn. Magn. Mater. 139, 263 (1995). 